\title{
Classification of wheat kernels infected with fungi of the genus Fusarium using discriminative classifiers and neural networks
}

\author{
Ewa Ropelewska ${ }^{1^{*}}$ \\ ${ }^{1}$ University of Warmia and Mazury in Olsztyn, Faculty of Engineering, Department of Systems Engineering, Heweliusza 14, 10-718 \\ Olsztyn, Poland. "Corresponding author (ewa.ropelewska@uwm.edu.pl).
}

Received: 21 August 2018; Accepted: 12 November 2018; doi:10.4067/S0718-58392019000100048

\begin{abstract}
Fusarium head blight (FHB) compromises the processing suitability and nutritional value of grain, and it causes significant crop losses. The aim of the study was to develop models for the classification of wheat (Triticum aestivum L.) kernels infected with fungi and healthy wheat kernels. Wheat kernels were classified with the use of Decision Tree, Rule-based, Bayes, Lazy, Meta and Function classifiers, as well as multilayer perceptron (MLP), radial basis function (RBF) and probabilistic neural networks (PNN). Twenty textures were selected from RGB, Lab, XYZ colour spaces each, for every wheat variety and each kernel side. Accuracy ranged from $82 \%$ for the dorsal side of kernels for Naive Bayes and IBk classifiers to $100 \%$ for the ventral side of kernels for IBk, FLDA and Naive Bayes classifiers. Classification accuracy was highest in the model based on texture attributes from Lab colour space. The final model of 20 attributes from Lab colour space was applied to a set of kernels from all wheat varieties, analysed on the ventral side. The accuracy of the classification model ranged from $94 \%$ to $98 \%$, depending on the applied classifier. The models developed with the use of neural networks were characterised by overall classification accuracy of above 99\% for MLP networks, above 96\% for RBF networks and above $97 \%$ for PNN. The developed models indicate that analyses should be performed on the ventral side of kernels based on textures from Lab colour space.
\end{abstract}

Key words: Discrimination, fungal infection, neural networks, textures, Triticum aestivum, wheat kernels.

\section{INTRODUCTION}

Wheat (Triticum aestivum L.) grain is a major global commodity which is used in the food processing industry to produce bread, biscuits, cakes, pasta, breakfast cereals, alcoholic beverages and animal feed (Dal-Pastro et al., 2016; Schmidt et al., 2016). Fungal contamination of wheat grain, in particular infections caused by fungi of the genus Fusarium, poses a significant problem. Fusarium head blight (FHB) compromises the processing suitability and nutritional value of grain, and it causes significant crop losses (Schmidt et al., 2016). Fusarium fungi also produce mycotoxins such as trichothecenes and zearalenone. Group B trichothecenes include deoxynivalenol (DON) and its acetylated derivatives (3AcDON, 15AcDON), nivalenol (NIV) and fusarenon X (FUS-X) (Kulik et al., 2015; Suproniene et al., 2016). Grain containing toxic fungal metabolites (mycotoxins) poses a health hazard (Schmidt et al., 2016). Therefore, infected grain has to be identified before it is processed into food and feed (Barbedo et al., 2015). Infected kernels are most commonly identified with the use of molecular, microscopic and microbial culture methods which are expensive, time consuming, laborious and require mycological expertise (Polder et al., 2005; Chelladurai et al., 2010; Levasseur-Garcia, 2012). Fungi can be identified based on the presence of mycotoxins in infected material. Mycotoxins are determined with the use of several methods and devices, including gas-chromatography (GC), high-performance liquid chromatography (HPLC), liquid chromatographymass spectrometry (LC/MS), enzyme-linked immunosorbent assay (ELISA), lateral flow devices (LFD), fluorescence 
polarization immunoassay (FPIA), infrared (IR) spectroscopy, electrical, optical and acoustic biosensors, and molecularly imprinted polymers (MIP). However, these methods require expensive equipment, complex sample preparation procedures and a relatively long time of analysis. Selected methods are characterized by low selectivity. Analytical methods for the identification of mycotoxins in grain should involve an extraction step, a clean-up step to eliminate interferences from the extract and to concentrate the analyte, and a final identification step (Pascale, 2009; Meneely et al., 2011). Also, the presence of fungi can be identified based on determination of the ergosterol content in grain (Ropelewska, 2018a). However, rapid, non-destructive and reliable methods for detecting infected kernels are required.

Digital image analyses based on colour descriptors were conducted by Jirsa and Polisenska (2011) to distinguish healthy and infected kernels and by Fox et al. (2004) to identify the discoloration of kernels caused by fungal sporodochia. There is a general scarcity of published information about texture attributes in the classification of infected and healthy wheat kernels. The grain of various cereal species and varieties was discriminated in digital image analyses based on their texture attributes. Majumdar and Jayas (2000) carried out a discriminant analysis based on 25 textural features, including 10 features from the grey level co-occurrence matrix (GLCM), 12 features from the grey level run length matrix (GLRM), and three grey level features. Textures extracted from colour channels R (red), G (green), B (blue) were applied. In a classification model based on 15 most significant textural features, mean training accuracy was $92.9 \%$ and mean testing accuracy was $92.0 \%$. These results accounted for $87.0 \%, 95.7 \%, 100.0 \%, 100.0 \%$ and $81.8 \%$ training accuracies and 85.2\%, 98.2\%, 100.0\%, 100.0\% and 76.3\% testing accuracies for Canada Western Red Spring (CWRS) wheat, Canada Western Amber Durum (CWAD) wheat, barley, oats and rye, respectively. Zapotoczny $(2011 ; 2014)$ identified wheat grain varieties based on textural features from R, G, B, Y, S, U, and V channels. The analysed wheat varieties were classified with $100 \%$ accuracy based on the textural attributes of single kernels and bulk grain. Guevara-Hernandez and Gomez-Gil (2011) determined 99 features in single kernels, including 21 morphological, 6 optical and 72 textural features. Wheat grain was evaluated with the use of models based on individual features and a combination of morphological, optical and textural features. The highest discrimination accuracy (above 99\%) was found in a model composed of features from all three groups. The average GLCM texture shifted by $90^{\circ}$ was the most important feature for classification.

The aim of the study was to develop models for the classification of wheat kernels infected with fungi and healthy wheat kernels. Textures with the highest discriminative power were selected, and the side of the kernel (ventral or dorsal) which provided more accurate results was determined. The results were used to develop a model which was characterised by high classification accuracy and was independent of the analysed wheat varieties.

\section{MATERIALS AND METHODS}

The study was performed on the grain of four wheat varieties. Grain samples of $5 \mathrm{~kg}$ each were obtained from farms and grain suppliers in various regions of Poland. Kernels infected with fungi of the genus Fusarium (pink, chalky white, shrivelled) and healthy kernels were selected from bulk samples.

\section{Image analysis}

Images were acquired with the use of the Epson Perfection 4490 Photo flatbed scanner (Seiko Epson Corp., Suwa, Nagano, Japan) and the SilverFast Ai Studio Epson v6.6.1r6 scanning application (LaserSoft Imaging, Kiel, Germany). Kernels were measured with a calliper. Individual kernels (infected and healthy) were scanned on both sides (ventral and dorsal) at a resolution of $500 \times 700$ pixels, $1200 \mathrm{dpi}$, tiff format. A total of 200 images, including 100 images of infected kernels and 100 images of healthy kernels, were acquired for each variety and each side. The images were analysed using MaZda v. 4.6 software (Łódź University of Technology, Institute of Electronics, Łódź, Poland) which supports image processing and quantitative analyses of textural features based on histogram, gradient, run-length matrix, co-occurrence matrix, an autoregressive model and Haar wavelet transform (Szczypinski et al., 2009). Textural features were computed from colour channels R, G, B, U, V, H, S, V, X, Y, Z, $\mathrm{L}, \mathrm{a}, \mathrm{b}$ and analyses were performed separately for colour models.

\section{Statistical analysis}

Wheat kernels were classified as infected or healthy with the use of classifiers and neural networks. Firstly, the classification was performed with the use of the WEKA 3.9 application (Machine Learning Group, University of Waikato, Hamilton, 
New Zealand) (Bouckaert et al., 2016). Wheat kernels were classified with the use of Decision Tree, Rule-based, Bayes, Lazy, Meta and Function classifiers by 10 -fold cross-validation. Attributes were selected to reduce the number of variables and shorten analysis time. The Ranker method with the OneR attribute evaluator was chosen. The selected method was characterised by the highest classification accuracy. In the first step, from the total set of attributes (textural features), 20 features with the highest discriminative power were selected from each colour model for each kernel side (ventral and dorsal) and each wheat variety. Twenty texture attributes were selected for discriminant analysis because the number of infected (100) and healthy (100) kernels of each wheat variety (a total of 200 kernels) was at least ten times greater than the number of attributes (20 textures). Kernels were classified separately in each colour model. Three colour models, which provided the highest classification accuracies were selected for further analyses. In the second stage of research, kernels were classified with the use of models containing textures from all colour spaces. For this purpose, seven textural features with the highest discriminative power were selected from the previously chosen group of 20 textures from each of the three selected colour spaces and combined into a single classification model, separately for each wheat variety. The models contained 21 attributes. In the third stage of the experiment, model incorporating the selected 21 textures (7 from RGB, Lab and XYZ colour models each) was developed for all wheat varieties. In the fourth stage, textures with the highest classification accuracy, chosen in the first stage of the experiment, were used to develop a model for testing a single set of kernels from all wheat varieties. This procedure was adopted to build a model independent of wheat variety. In the following stage, the model developed in the WEKA application was used to classify infected and healthy wheat kernels with the use of neural networks. The analyses based on multilayer perceptron (MLP) and radial basis function (RBF) neural networks were carried out in Statistica 12.0 software (StatSoft Inc., Tulsa, Oklahoma, USA; 2013). The analysed set was divided into three data sets in two ways: $70 \%$ for learning, $15 \%$ for testing and $15 \%$ for validation, as well as $80 \%$ for learning, $10 \%$ for testing and $10 \%$ for validation. The MATLAB 8.3 R2014a application (MathWorks, Inc., Natick, Massachusetts, USA) was used for analyses based on a probabilistic neural network (PNN). The set was divided into two data sets: $80 \%$ for learning and $20 \%$ for testing.

\section{RESULTS}

The classification models based on 20 variables with the highest discriminative power for RGB, Lab and XYZ colour models were built to discriminate healthy wheat kernels and kernels infected with fungi. Kernels from each wheat variety were analysed separately on ventral and dorsal sides. Classification accuracies ranged from $82 \%$ to $100 \%$ (Table 1). In analyses of the dorsal side of variety 1 kernels, Naive Bayes and IBk classifiers were characterised by

Table 1. Classification accuracy of infected and healthy wheat kernels based on 20 textures selected from each colour model, for kernels of each wheat variety, positioned on the ventral and dorsal side.

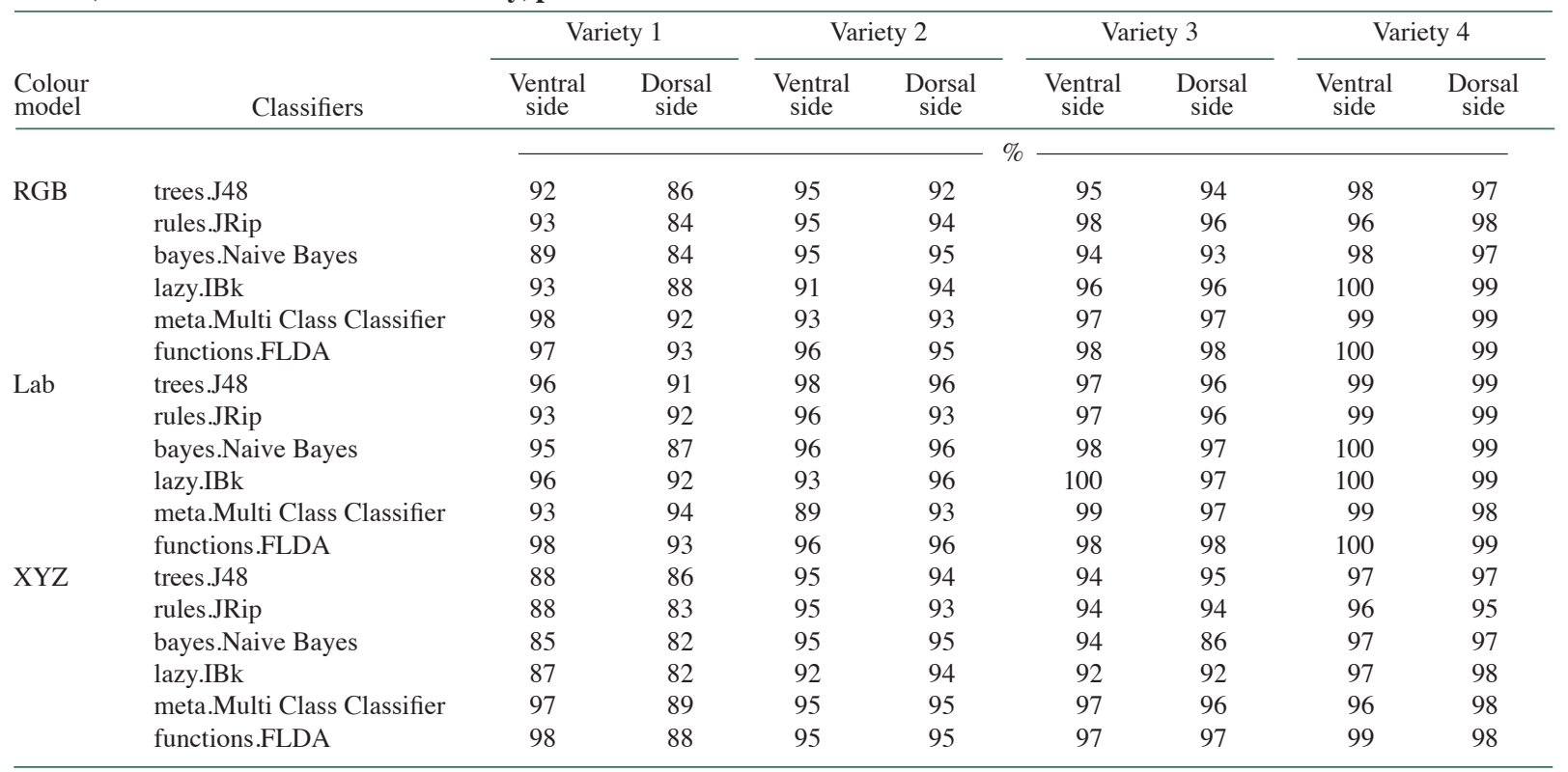


lower classification accuracy (82\%). Classification accuracy reached 100\% when the ventral side of kernels of two varieties ( 3 and 4 ) was analysed with IBk, FLDA and Naive Bayes classifiers. The above implies that all infected kernels were correctly assigned to the "infected" class and all healthy kernels to the "healthy" class. In dorsal side analyses, none of the tested varieties or classifiers produced $100 \%$ accuracy. The obtained results could indicate that visible symptoms of fungal infection differ on each side of the kernel. Classification accuracy was higher on the ventral than on the dorsal side. Symptoms of fungal infection were more visible on the ventral side of kernels. The crease on wheat kernels could facilitate the growth and development of fungi. Wheat variety also influenced the discrimination of infected and healthy kernels. Classification accuracy was highest for variety 4 (96\%-100\% for the ventral side and 95\%-99\% for the dorsal side) and lowest for variety 1 (85\%-98\% for the ventral side and $82 \%-94 \%$ for the dorsal side). The observed differences could be attributed to variations in the physical properties of kernels of different wheat varieties, agronomic methods and climatic conditions. In this study, grain samples were collected from various Polish regions to account for local differences. The colour model influenced the discrimination of infected and healthy kernels (Table 1). The model based on textures from Lab colour space delivered the most satisfactory results. Classification accuracy was $87 \%-100 \%$, and in most cases, the results were much higher than in RGB and XYZ models. The XYZ model was characterised by the lowest classification accuracy of 82\%-99\%. Classification accuracy did not reach $100 \%$ in models based on textures from XYZ colour space.

In the following stage of the study, kernels were classified with the use of models containing 21 attributes, including seven textures from each colour space (RGB, Lab, XYZ). In previous analyses, classification accuracies were higher for the ventral side (Table 1), therefore, only this side was considered in this stage. Kernels of each variety were classified separately. The obtained results are presented in Table 2. Classification accuracy ranged from $93 \%$ to $100 \%$. The accuracy of classification into infected and healthy kernels was influenced by variety. Classification accuracy was highest in variety 4 at 99\%-100\% and lowest in variety 1 at 93\%-98\%. Naive Bayes, IBk and Multi Class classifiers discriminated kernels with $100 \%$ accuracy.

Attempts were also made to develop a model that accurately discriminates infected and healthy kernels independently of variety. Initially, the model was built based on 21 textures ( 7 from RGB, Lab and XYZ colour models each) for kernels of each variety positioned on the ventral side. Classification accuracy ranged from $91 \%$ for Naive Bayes to $96 \%$ for IBk, Multi Class and FLDA classifiers (Table 3).

Table 2. Classification accuracy of infected and healthy wheat kernels based on the selected 21 textures (seven from RGB, Lab and XYZ colour models) for ventral side of each varieties.

\begin{tabular}{lccccc}
\hline Classifiers & Variety 1 & Variety 2 & Variety 3 & Variety 4 \\
\cline { 2 - 3 } \cline { 2 - 3 } trees.J48 & 94 & 96 & & 97 & 99 \\
rules.JRip & 94 & 96 & 96 & 99 \\
bayes.Naive Bayes & 93 & 96 & 99 & 100 \\
lazy.IBk & 94 & 95 & 99 & 100 \\
meta.Multi Class Classifier & 98 & 98 & & 98 & 100 \\
functions.FLDA & 96 & 96 & & 98 & 99 \\
\hline
\end{tabular}

Table 3. Classification accuracy of infected and healthy wheat kernels based on 21 textures (seven from RGB, Lab and XYZ colour models each) for kernels from a set containing all varieties, positioned on the ventral side.

\begin{tabular}{lc}
\hline \multicolumn{1}{c}{ Classifier } & All varieties \\
\hline & $\%$ \\
trees.J48 & 95 \\
rules.JRip & 95 \\
bayes.Naive Bayes & 91 \\
lazy.IBk & 96 \\
meta.Multi Class Classifier & 96 \\
functions.FLDA & 96 \\
\hline
\end{tabular}


Attempts were also made to increase the accuracy of the classification model. Only textures from colour space which produced the highest classification accuracy were used in the model (Table 1). The model included 20 attributes from Lab colour space, and it was applied to a set containing all varieties. Individual kernels were analysed on the ventral side where symptoms of fungal infection were more visible. Classification accuracy was high at 94\%-98\%, depending on the applied classifier (Table 4). Classifier IBk produced the most satisfactory results. The scatterplots of selected textures are presented in Figure 1. The textural features of infected and healthy kernels differ considerably in value. The values of attribute LHPerc90 (Figure 1A) were high in infected kernels, and the values of bHMean (Figure 1B) were high in healthy kernels.

The model (Table 4) was applied to discriminate kernels with the use of neural networks. The model contained 20 textures from Lab colour space, for kernels positioned on the ventral side and a set consisting of all varieties. The results of classification with the use of MLP, RBF neural networks are presented in Table 5. The highest accuracies were obtained when the data set was divided as follows: $70 \%$ for learning, $15 \%$ for testing and $15 \%$ for validation. The overall classification accuracy achieved with MLP networks was 99.64\%, where infected kernels were correctly classified in $99.63 \%$ cases, and healthy kernels in $99.65 \%$ cases. When the data set was divided into a learning set (80\%), a testing set (10\%) and a validation set (10\%), the overall classification accuracy was highest for MLP (99.53\%) and RBF (96.25\%) networks. When the data set was divided into a learning set (80\%) and a testing set (20\%), the overall classification accuracy for a probabilistic neural network (PNN) was 97.22\%. Infected kernels were correctly discriminated in $94.44 \%$ cases, and healthy kernels - in $100 \%$ cases.

Table 4. Classification accuracy of infected and healthy wheat kernels based on 20 textures from the Lab colour model for kernels from a set containing all varieties, positioned on the ventral side.

\begin{tabular}{lc}
\hline \multicolumn{1}{c}{ Classifier } & All varieties \\
\hline & $\%$ \\
trees.J48 & 97 \\
rules.JRip & 96 \\
bayes.Naive Bayes & 94 \\
lazy.IBk & 98 \\
meta.Multi Class Classifier & 97 \\
functions.FLDA & 97 \\
\hline
\end{tabular}

Figure 1. Scatterplots of attributes LHPerc90 (A) and bHMean (B) in infected (red) and healthy kernels (blue).
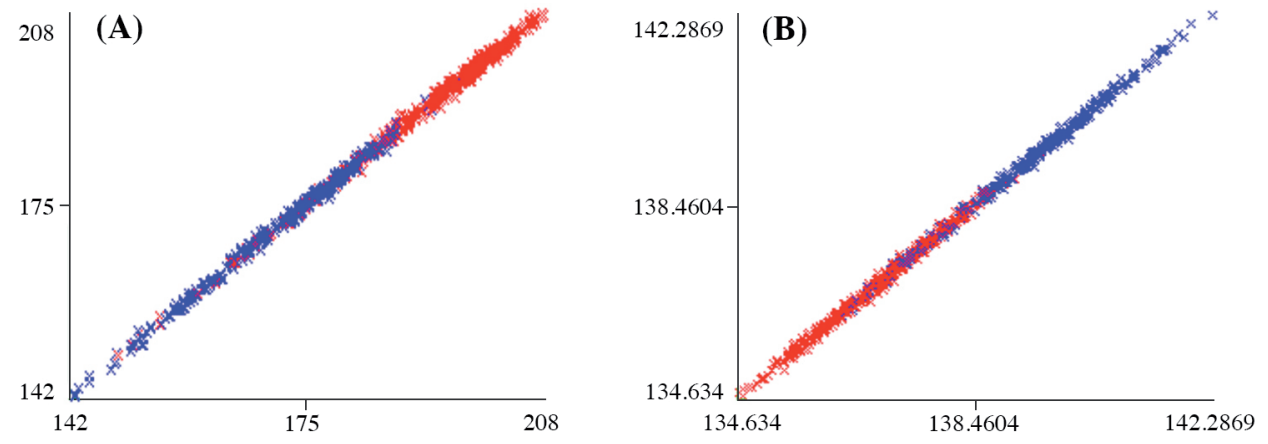
Table 5. Classification accuracy based on 20 textures from the Lab colour model for kernels from a set containing all varieties, positioned on the ventral side, determined with the use of neural networks.

\begin{tabular}{|c|c|c|c|c|}
\hline \multirow[b]{2}{*}{ Neural network } & \multirow{2}{*}{$\begin{array}{c}\text { Classification } \\
\text { accuracy }\end{array}$} & \multicolumn{3}{|c|}{ Experimental group } \\
\hline & & Infected & Healthy & Total \\
\hline \multicolumn{5}{|c|}{ Data set division: $70 \%$ for learning, $15 \%$ for testing, and $15 \%$ for validation } \\
\hline \multirow[t]{2}{*}{ MLP 20-12-2 } & Correct $(\%)$ & 99.63 & 99.65 & 99.64 \\
\hline & Incorrect $(\%)$ & 0.37 & 0.35 & 0.36 \\
\hline \multirow[t]{2}{*}{ MLP 20-10-2 } & Correct $(\%)$ & 99.26 & 99.30 & 99.29 \\
\hline & Incorrect $(\%)$ & 0.74 & 0.69 & 0.71 \\
\hline \multirow[t]{3}{*}{ MLP 20-13-2 } & Correct $(\%)$ & 99.26 & 98.96 & 99.11 \\
\hline & Incorrect (\%) & 0.74 & 1.04 & 0.89 \\
\hline & \multicolumn{4}{|c|}{ Data set division: $80 \%$ for learning, $10 \%$ for testing, and $10 \%$ for validation } \\
\hline \multirow[t]{2}{*}{ MLP 20-11-2 } & Correct $(\%)$ & 99.36 & 99.70 & 99.53 \\
\hline & Incorrect $(\%)$ & 0.64 & 0.30 & 0.47 \\
\hline \multirow[t]{2}{*}{ MLP 20-18-2 } & Correct $(\%)$ & 98.07 & 98.18 & 98.13 \\
\hline & Incorrect $(\%)$ & 1.93 & 1.82 & 1.87 \\
\hline \multirow[t]{2}{*}{ RBF 20-21-2 } & Correct $(\%)$ & 99.68 & 93.01 & 96.25 \\
\hline & Incorrect $(\%)$ & 0.32 & 6.99 & 3.75 \\
\hline
\end{tabular}

\section{DISCUSSION}

The obtained results have expanded our knowledge about the applicability of image analysis for the identification of fungal infection of cereal grain. In the available literature, there are several reports on the evaluation of Fusariuminfected kernels with the use of physical parameters. Jirsa and Polisenska (2011) used image analysis based on geometric parameters, such as: length, width, area and perimeter, as well as colour descriptors: R (Red), G (Green), B (Blue) and $\mathrm{H}$ (Hue) of infected and healthy wheat kernels. The authors obtained a high accuracy reaching $85 \%$ in infected and healthy wheat kernels classified based on model containing colour descriptors. However, they reported that geometric parameters were not specific to distinguish Fusarium-infected and healthy kernels. Menesatti et al. (2009) applied elliptic Fourier analysis to extract shape information and discriminate the wheat kernels in three classes: healthy, shrivelled and chalky. The total accuracy of classification of these classes for kernels infected with Fusarium culmorum was $68.4 \%$ and Fusarium graminearum was $56.9 \%$.

According to literature data, discrimination models based on textural features allowed to increase the classification accuracy of infected and healthy cereal kernels. Ropelewska and Zapotoczny (2018) reported the accuracy of 76\%$100 \%$ for classification models of infected and healthy wheat kernels including the textures from flatbed scanner and hyperspectral images. For images acquired with a hyperspectral imaging system at different wavelengths, classification accuracy of 76\%-98\% was observed. In the case of images acquired with a flatbed scanner, the selected textures from colour channels: L* (Lightness), R (Red) and Y (Luminance) were considered. The high classification accuracy of 95\%$100 \%$ was found. The results obtained in present paper supplemented this knowledge, by considering a greater number of another varieties of wheat and using another classification model based on textures from RGB, Lab or XYZ colour spaces. Furthermore, in addition to models built with the use of discriminative classifiers, the models using neural networks were developed. The usefulness of image analysis based on texture attributes for the identification of fungal infection in kernels, which are demonstrated in present paper for wheat, was also reported by Ropelewska (2018b) for barley. Ropelewska (2018b) observed high accuracy of classification up to $100 \%$. Similar to the results of present studies for wheat, Ropelewska (2018b) found that for barley the most satisfactory results were observed for models based on textures from Lab colour space and the lowest accuracies were for textures selected from XYZ model. The obtained results are the basis for further research on infection of cereal kernels. It can be used for investigation of the quantity of fungal DNA in kernels based on texture features. 


\section{CONCLUSIONS}

The results of this study indicate that the visible symptoms of fungal infection differ on each side of a kernel. Fungal symptoms were more visible on the ventral than the dorsal side. The presence of a crease on the ventral side of wheat kernels could facilitate the growth and development of fungi. Wheat variety also influenced the discrimination of infected and healthy kernels. The observed differences could be attributed to variations in the physical properties of kernels from different wheat varieties, agronomic methods and climatic conditions. Classification accuracy was determined by the group of textures included in the model. Textures from Lab colour space produced the most satisfactory results. A model which was characterised by high classification accuracy and was independent of the analysed wheat varieties was developed based on selected textures from Lab space for kernels positioned on the ventral side. Classification accuracy was high when both classifiers and neural networks were used. The results can be used in further studies investigating the quantity of fungal DNA in kernels based on texture features. The developed models indicate that analyses should be performed on the ventral side of kernels based on textures from Lab colour space.

\section{ACKNOWLEDGEMENTS}

This study was conducted as part of a comprehensive research project financed by the University of Warmia and Mazury in Olsztyn (grant Nr 16.620.014-300).

\section{REFERENCES}

Barbedo, J.G.A., Tibola, C.S., and Fernandes, J.M.C. 2015. Detecting Fusarium head blight in wheat kernels using hyperspectral imaging. Biosystems Engineering 131:65-76.

Bouckaert, R.R., Frank, E., Hall, M., Kirkby, R., Reutemann, P., Seewald, A., et al. 2016. WEKA Manual for Version 3-9-1. University of Waikato, Hamilton, New Zealand.

Chelladurai, V., Jayas, D.S., and White, N.D.G. 2010. Thermal imaging for detecting fungal infection in stored wheat. Journal of Stored Products Research 46:74-179.

Dal-Pastro, F., Facco, P., Bezzo, F., Zamprogna, E., and Barolo, M. 2016. Data-driven modeling of milling and sieving operations in a wheat milling process. Food and Bioproducts Processing 99:99-108.

Fox, G., Sulman, M., Johnson, V., Young, K., and Inkerman, A. 2004. Strategies for breeding against barley grain colour defects . In Fischer, T., Turner, N., Angus, J., McIntyre, L., Robertson, M., Borrell, A., et al. (eds.) Proceedings of the 4th International Crop Science Congress, Brisbane, Australia. Regional Institute Ltd., Gosford, Australia.

Guevara-Hernandez, F., and Gomez-Gil, J. 2011. A machine vision system for classification of wheat and barley grain kernels . Spanish Journal of Agricultural Research 9(3):672-680.

Jirsa, O., and Polisenska, I. 2011. Identification of Fusarium damaged wheat kernels using image analysis. Acta Universitatis Agriculturae et Silviculturae Mendelianae Brunensis 59:125-130.

Kulik, T., Ostrowska, A., Buśko, M., Pasquali, M., Beyer, M., Stenglein, S., et al. 2015. Development of an FgMito assay: A highly sensitive mitochondrial based qPCR assay for quantification of Fusarium graminearum sensu stricto. International Journal of Food Microbiology 210:16-23.

Levasseur-Garcia, C. 2012. Infrared spectroscopy applied to identification and detection of microorganisms and their metabolites on cereals (corn, wheat, and barley). p. 185-196. In Aflakpui, D.G. (ed.) Agricultural science. InTech Europe, Rijeka, Croatia.

Majumdar, S., and Jayas, D.S. 2000. Classification of cereal grains using machine vision: III. Texture models. Transactions of the ASAE 43(6):1681-1687.

Meneely, J.P., Ricci, F., van Egmond, H.P., and Elliott, C.T. 2011. Current methods of analysis for the determination of trichothecene mycotoxins in food. Trends in Analytical Chemistry 30(2):192-203.

Menesatti, P., Antonucci, F., Costa, C., Santori, A., Niciarelli, I., and Infantino, A. 2009. Application of morphometric image analysis system to evaluate the incidence of Fusarium head blight wheat infected kernels. Proceedings of Bornimer Agrartechnische Berichte-Heft, , Potsdam, Berlin, Germany, 157-161.

Pascale, M.N. 2009. Detection methods for mycotoxins in cereal grains and cereal products. Proceedings for Natural Sciences/ Matica Srpska 117:15-25.

Polder, G., van der Heijden, G.W.A.M., Waalwijk, C., and Young, I.T. 2005. Detection of Fusarium in single wheat kernels using spectral imaging. Seed Science and Technology 33:655-668.

Ropelewska, E. 2018a. Relationship of thermal properties and ergosterol content of barley grains. Journal of Cereal Science 79:328-334. 
Ropelewska, E. 2018b. The application of computer image analysis based on textural features for the identification of barley kernels infected with fungi of the genus Fusarium. Agricultural Engineering 22(3):49-56.

Ropelewska, E., and Zapotoczny, P. 2018. Classification of Fusarium-infected and healthy wheat kernels based on features from hyperspectral images and flatbed scanner images: a comparative analysis. European Food Research and Technology 244(8):1453-1462.

Schmidt, M., Horstmann, S., De Colli, L., Danaher, M., Speer, K., Zannini, E., et al. 2016. Impact of fungal contamination of wheat on grain quality criteria. Journal of Cereal Science 69:95-103.

Suproniene, S., Sakalauskas, S., Stumbriene, K., Zvirdauskiene, R., and Svegzda, P. 2016. Variances in trichothecene chemotype distribution in Lithuanian wheat grain and within pure culture Fusarium graminearum isolated from the same grain samples. European Journal of Plant Pathology 144:371-381.

Szczypinski, P.M., Strzelecki, M., Materka, A., and Klepaczko, A. 2009. MaZda - A software package for image texture analysis. Computer Methods and Programs in Biomedicine 94(1):66-76.

Zapotoczny, P. 2011. Discrimination of wheat grain varieties using image analysis and neural networks. Part I. Single kernel texture. Journal of Cereal Science 54:60-68.

Zapotoczny, P. 2014. Discrimination of wheat grain varieties using image analysis and multidimensional analysis texture of grain mass. International Journal of Food Properties 17:139-151. 